\title{
ALMA photometry of extragalactic radio sources
}

\author{
M. Bonato ${ }^{\oplus, 1,2 \star}$ E. Liuzzo, ${ }^{1}$ D. Herranz,${ }^{3}$ J. González-Nuevo ${ }^{\oplus},{ }^{4}$ L. Bonavera ${ }^{\oplus},{ }^{4}$ \\ M. Tucci, ${ }^{5}$ M. Massardi, ${ }^{1}$ G. De Zotti, ${ }^{2}$ M. Negrello ${ }^{6}$ and M. A. Zwaan ${ }^{7}$ \\ ${ }^{1}$ INAF - Istituto di Radioastronomia, and Italian ALMA Regional Centre, Via Gobetti 101, I-40129 Bologna, Italy \\ ${ }^{2}$ INAF - Osservatorio Astronomico di Padova, Vicolo Osservatorio 5, I-35122 Padova, Italy \\ ${ }^{3}$ Instituto de Física de Cantabria (CSIC-Universidad de Cantabria), Avda. de los Castros s/n, E-39005 Santander, Spain \\ ${ }^{4}$ Departamento de Física, Universidad de Oviedo, C. Federico García Lorca 18, E-33007 Oviedo, Spain \\ ${ }^{5}$ Département de Physique Théorique and Center for Astroparticle Physics, Université de Genève, 24, Quai E. Anserment, 1211, Genève, Switzerland \\ ${ }^{6}$ School of Physics and Astronomy, Cardiff University, The Parade, Cardiff CF24 3AA, UK \\ ${ }^{7}$ European Southern Observatory, Karl-Schwarzschild-Str. 2, D-85748 Garching, Germany
}

Accepted 2019 February 1. Received 2019 January 30; in original form 2018 December 10

\begin{abstract}
We present a new catalogue of ALMA (Atacama Large Millimeter/submillimeter Array) observations of 3364 bright, compact radio sources, mostly blazars, used as calibrators. These sources were observed between 2011 May and 2018 July, for a total of 47115 pointings in different bands and epochs. We have exploited the ALMA data to validate the photometry given in the new Planck multifrequency Catalogue of Non-Thermal sources (PCNT), for which an external validation was not possible so far. We have also assessed the positional accuracy of Planck catalogues and the PCNT completeness limits, finding them to be consistent with those of the Second Planck Catalogue of Compact Sources. The ALMA continuum spectra have allowed us to extrapolate the observed radio source counts at $100 \mathrm{GHz}$ to the effective frequencies of ALMA bands 4, 6, 7, 8, and 9 (145, 233, 285, 467, and $673 \mathrm{GHz}$, respectively), where direct measurements are scanty, especially at the three highest frequencies. The results agree with the predictions of the Tucci et al. model C2Ex, while the model C2Co is disfavoured.
\end{abstract}

Key words: galaxies: active - galaxies: statistics - radio continuum: galaxies - submillimetre: galaxies.

\section{INTRODUCTION}

Although a substantial progress on the characterization of millimetre $(\mathrm{mm})$ and submillimetre (submm) properties of extragalactic radio sources has been made in recent years mainly thanks to surveys with the Wilkinson Microwave Anisotropy Probe (Bennett et al. 2013), the Planck satellite (Planck Collaboration XXVI 2016; Planck Collaboration Int. LIV 2018), the South Pole Telescope (SPT; Mocanu et al. 2013), and the Atacama Cosmology Telescope (ACT; Marsden et al. 2014), the available information is still limited.

However, an unprecedented amount of multifrequency and multiepoch photometric data on radio sources in this spectral region is being provided by the Atacama Large Millimeter/submillimeter Array (ALMA). This is because ALMA uses mm/submm bright compact radio sources as calibrators to fix the flux density scale, to determine the bandpass response, and to calibrate amplitude and phase of the visibilities of the science targets. Observations of calibrator sources (mainly point-like sources) are made for every science project. Each calibrator is generally observed several times, in connection with different targets, on different days, in various bands and array configurations.

Bonato et al. (2018) have published a catalogue of ALMA flux density measurements of 754 calibrators for a total of 16263 observations in different bands and epochs (ALMACAL catalogue). These flux densities were uniformly measured from a collection of ALMA images, thus obtaining robust measurements for both resolved and non-resolved sources. The calibration and imaging analyses are described in Bonato et al. (2018). Almost all sources ( $\sim 97$ per cent) were found to be blazars.

Combining such catalogue with the 'ALMA Calibrator Source Catalogue' (ACSC), ${ }^{1}$ we have collected ALMA observations for 3364 bright, (mostly) compact radio sources. These sources were observed between 2011 May and 2018 July, for a total of 47115 observations in different bands and epochs. We have 25907 observations of 3310 different sources in band $3(84-116 \mathrm{GHz})$, 671 observations of 171 sources in band $4(125-163 \mathrm{GHz}), 8467$ observations of 885 sources in band 6 (211-275 GHz), 11415 observations of 2201 sources in band 7 (275-373 GHz), 394 observations 
Table 1. Example of the catalogue content. The full catalogue is available online and on the website of the Italian ARC (http://arc.ia2.inaf.it).

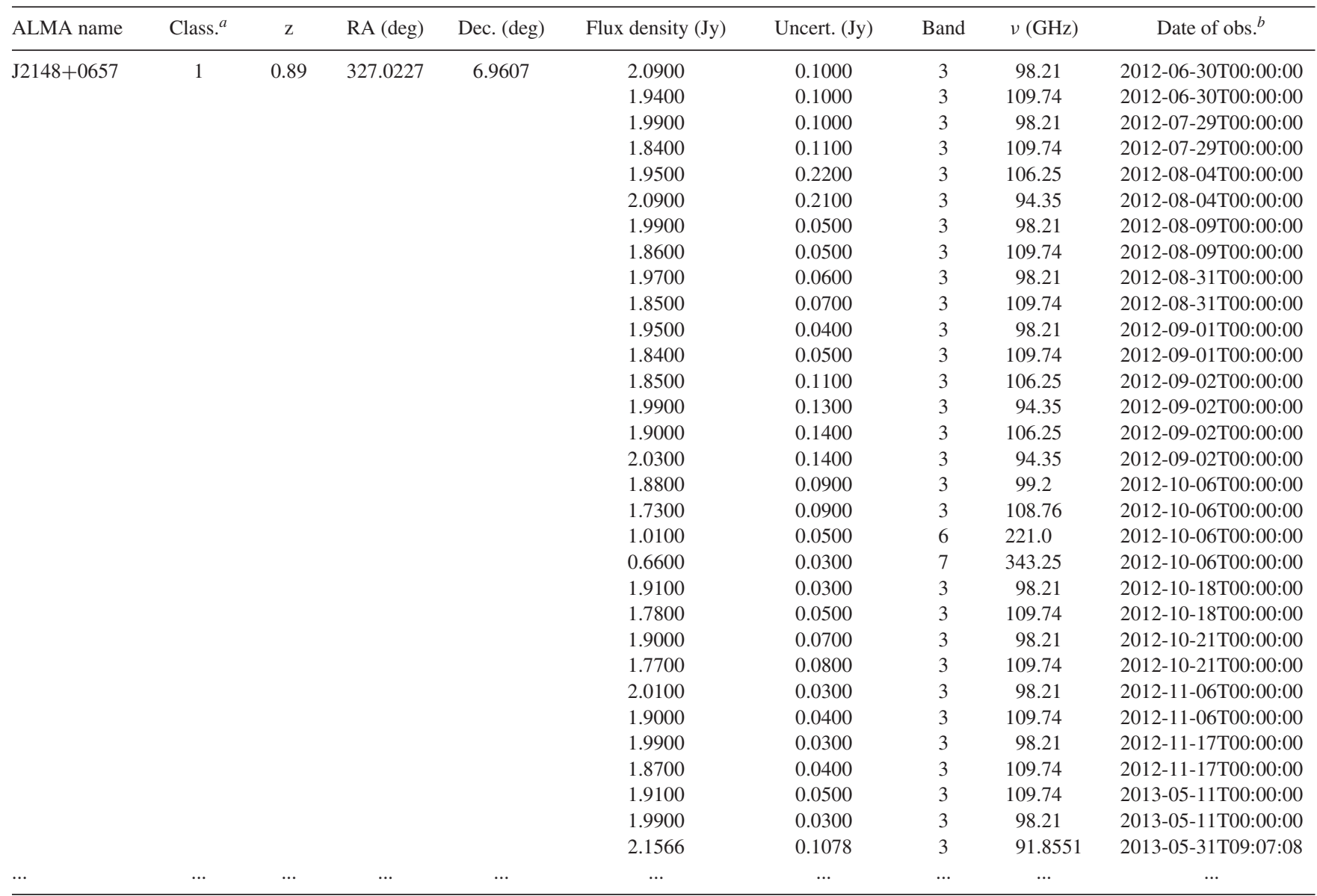

Notes: ${ }^{\text {a }}$ Classification: 1 = Flat-spectrum radio quasar (FSRQ); 2 = BL Lac; 3 = BL Lac-galaxy dominated; 4 = Blazar uncertain type;

$5=$ BL Lac candidate; $6=$ Steep spectrum; and $7=$ Uncertain.

${ }^{b}$ Observing date and time in the ISO standard format [YYYY-MM-DDThh:mm:ss], UTC time. Observations taken from the

'ALMA Calibrator Source Catalogue', for which time information is not available, appear with time '00:00:00'.

of 88 sources in band $8(385-500 \mathrm{GHz}), 253$ observations of 59 sources in band $9(602-720 \mathrm{GHz})$, and 8 observations of 6 sources in band 10 (787-950 GHz).

The combined catalogue, referred to as the ALMA Calibrator Catalogue (ACC), contains the ALMA name of the source, its classification, redshift (when available), equatorial coordinates (J2000), flux density measurements with their uncertainties, effective observing frequency, date, and time of observations. One example of the information provided is in Table 1.

A search of the literature has yielded redshifts for 2245 sources (67 per cent). About 41 per cent (1391) of our sources are listed in the fifth edition of the Roma Multifrequency Catalogue of Blazars ${ }^{2}$ (BZCAT; Massaro et al. 2009) where they are classified into five sub-classes: FSRQs, BL Lacs, BL Lacs-galaxy dominated, Blazars of uncertain type, and BL Lac candidates. We have classified the remaining objects following Bonato et al. (2018).

The uncertainties on ALMA flux densities are essentially given by the calibration uncertainty, whose value is still being debated within the ALMA community. An accurate calibration is difficult to achieve due to variability of the emissive and absorptive properties of the Earth's atmosphere and to the lack of astronomical sources

\footnotetext{
${ }^{2}$ http://www.ssdc.asi.it/bzcat/
}

that could serve as accurate flux standards. Following Bonato et al. (2018), we adopt at 5 percent calibration uncertainty for ALMACAL sources. This value was found to be consistent with the median absolute differences among measurements in bands 3 and 6 within short timespans ( $30 \mathrm{~d}$ in the source frame), expected to be only weakly affected by variability (see section 4 of Bonato et al. 2018).

We have made a similar check on ACSC sources. We selected observations within $30 \mathrm{~d}$ in the source frame. For this timespan, we found median absolute differences of 6 per cent for bands 3 and 6 , and of 8 per cent for band 7. There are no multiple measurements within this timespan in bands 4,8 , and 9 , so that no estimates could be obtained. These somewhat larger uncertainties for ACSC compared to ALMACAL sources may be due to the fact that the flux densities of the latter sources were uniformly measured from a collection of ALMA images. The measured differences are however consistent with the uncertainties given in the ACSC, taking into account that outliers are to be expected since blazars show variability also on short time-scales. We have therefore adopted the ACSC uncertainties.

In this paper, we exploit the ACC catalogue for two purposes. In Section 2, we use ALMA photometry to validate the new flux density estimates presented in the Planck multifrequency Catalogue of Non-Thermal sources (PCNT; Planck Collaboration Int. LIV 


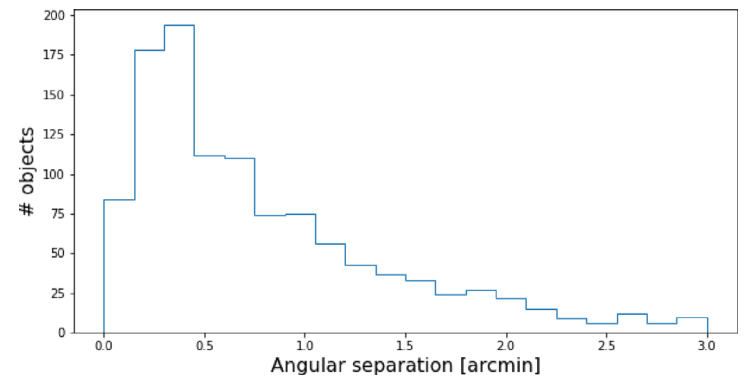

Figure 1. Distribution of angular separations between ALMA and Planck Collaboration XXVI (2016) positions of the 1120 sources resulting from the cross-match ( 3 arcmin search radius) between the ACC and the PCNT catalogues.

2018), and to assess the Planck completeness limits and positional accuracy. So far, only an internal validation was possible and was indeed performed.

Next, we exploit the multifrequency ALMA measurements to estimate the distribution of flux density ratios between band 3 and higher frequency bands. Such distributions allow us to extrapolate the observed $100 \mathrm{GHz}$ source counts to higher frequencies where direct measurements are quite limited or missing (Section 3). Finally, in Section 4, we present our main conclusions.

\section{VALIDATION OF THE PLANCK MULTIFREQUENCY CATALOGUE OF NON-THERMAL SOURCES}

The PCNT was built performing a multifrequency analysis using the 'Matrix Filters' (MTFX) methodology (see Herranz \& Sanz 2008; Herranz et al. 2009) at the positions of sources detected by the Mexican Hat Wavelet 2 algorithm (González-Nuevo et al. 2006; López-Caniego et al. 2006) in the full mission all-sky temperature maps $^{3}$ with a signal-to-noise ratio $(\mathrm{S} / \mathrm{N})$ larger than 3 at either 30 and $143 \mathrm{GHz}$. The MTFX yielded flux densities and uncertainties in all nine Planck channels. This multifrequency approach has made it possible to reach deeper detection limits, at given $\mathrm{S} / \mathrm{N}$, than the Second Planck Catalogue of Compact Sources (PCCS2; Planck Collaboration XXVI 2016) which contains sources detected in each frequency channel separately.

So far the MTFX photometry could be validated only by comparison with flux densities reported in the PCCS2, complemented with a statistical check made comparing the number counts of catalogued sources with models. But PCCS2 estimates for PCNT sources are generally missing above $217 \mathrm{GHz}$. Moreover, the amount of external data available to validate them was quite limited, particularly at $\mathrm{mm}$ and submm wavelengths.

To check the MTFX photometry up to high frequencies we crossmatched the ACC with the PCNT outside the GAL070 mask, i.e. excluding the $\simeq 30$ per cent of the sky more heavily contaminated by Galactic emissions. We used a search radius of 3 arcmin, more than a factor of 3 larger than the estimated positional uncertainties of Planck sources $(\lesssim 1$ arcmin, see tables 5 and 6 of Planck Collaboration XXVI 2016). As illustrated by Fig. 1, this positional uncertainty estimate is confirmed by the comparison between ALMA and Planck positions. The positional differences peak at $\sim 0.38$ arcmin and have a standard deviation $\sigma \simeq 0.64 \mathrm{arcmin}$. The distribution is however strongly asymmetric with an extended tail

${ }^{3}$ The time frame of these data is $\sim 2.5 \mathrm{yr}$. towards separations of a few arcminutes. This tail slightly decreases if sources below the PCCS2 90 percent completeness limits are excluded.

We found unique Planck counterparts with $\mathrm{S} / \mathrm{N} \geq 3$ for 1120 out of the 3364 ALMA calibrators. Specifically, we found 1069 counterparts (out of 3310 calibrators) at $100 \mathrm{GHz}, 100$ (out of 171) at $143 \mathrm{GHz}, 455$ (out of 885) at $217 \mathrm{GHz}$, and 439 (out of 2,201) at $353 \mathrm{GHz}$. Moreover, 60 sources with ALMA measurements in band 8 or 9 have Planck counterparts at $545 \mathrm{GHz}$. Obviously there is a large overlap among sources observed in the different ALMA bands.

The mean surface density of PCNT sources outside the GAL070 mask is $\simeq 0.04 \mathrm{deg}^{-2}$, so that the probability that a PCNT source lies by chance within 3 arcmin of an ALMA source is $\simeq 3 \times 10^{-4}$. Thus the expected number of spurious associations is $\simeq 1$, i.e. $\simeq 0.1$ per cent of Planck counterparts.

MTFX flux density estimates at 100, 143, 217, and $353 \mathrm{GHz}$ were compared to ALMA flux densities in bands 3, 4, 6, and 7, respectively. The small differences of effective frequencies between bands 3, 4, and 6 and the nearest Planck channels were neglected. Band 7 flux densities were converted to $353 \mathrm{GHz}$ with $S \propto v^{-0.45}$, using the mean spectral index of our sources between bands 6 and 8 . The Planck $545 \mathrm{GHz}$ channel is intermediate in frequency to ALMA bands 8 and 9. We have extrapolated ALMA flux densities in these bands to $545 \mathrm{GHz}$ adopting the mean spectral index of our ALMA sources between these bands, i.e. with $S \propto v^{-0.62}$. In any case, the extrapolations in frequency are quite small and the spectral indices are relatively flat so that the results are only weakly affected by chosen values.

For most sources, we have several ALMA observations in each band. The comparison with PCNT flux densities was made using the mean values and adopting the standard deviation, summed in quadrature with the calibration uncertainty, as an estimate of the uncertainty.

The results for Planck channels from 100 to $545 \mathrm{GHz}$ are illustrated by Fig. 2. At the faintest flux density levels, the MTFX photometry is affected by the Eddington bias (Eddington 1913; Hogg \& Turner 1998), which accounts for the increase of the Planck/ALMA flux density ratio with decreasing flux density. Above the PCCS2 90 per cent completeness limits (vertical dotted red lines), we find good consistency between the MTFX and the ALMA photometry. The large dispersion of flux density ratios (typically $\left.\sigma\left(\log \left(S_{\mathrm{PCNT}} / S_{\mathrm{ALMA}}\right)\right) \simeq 0.2\right)$ can be entirely ascribed to variability ${ }^{4}$ plus measurement uncertainties, mostly on the Planck side. In particular, the few sources in the brightest luminosity bin have most likely been caught by Planck in a flaring phase. ${ }^{5}$

\footnotetext{
${ }^{4}$ Blazars are known to be strongly variable and Planck and ALMA measurements are non-simultaneous. Planck flux densities are averages over the five full sky surveys with the high-frequency instrument from 2009 August 12 to 2012 January 11. ALMA observations are distributed between 2011 May and 2018 July.

${ }^{5}$ Variability affects the Planck/ALMA comparison at all flux density levels. In well-populated flux density bins its main effect is to increase the dispersion of flux density ratios. The highest luminosity bins, however, contain only a few sources whose mean flux density can be dominated by extreme outbursts increasing flux densities by factors of 10 or more. This is the simplest explanation of anomalously high Planck/ALMA ratios such as the one in the brightest bin at $545 \mathrm{GHz}$ of Fig. 2. This bin contains only two sources, measured with high S/N both by Planck and by ALMA, so that the difference must be real.
} 

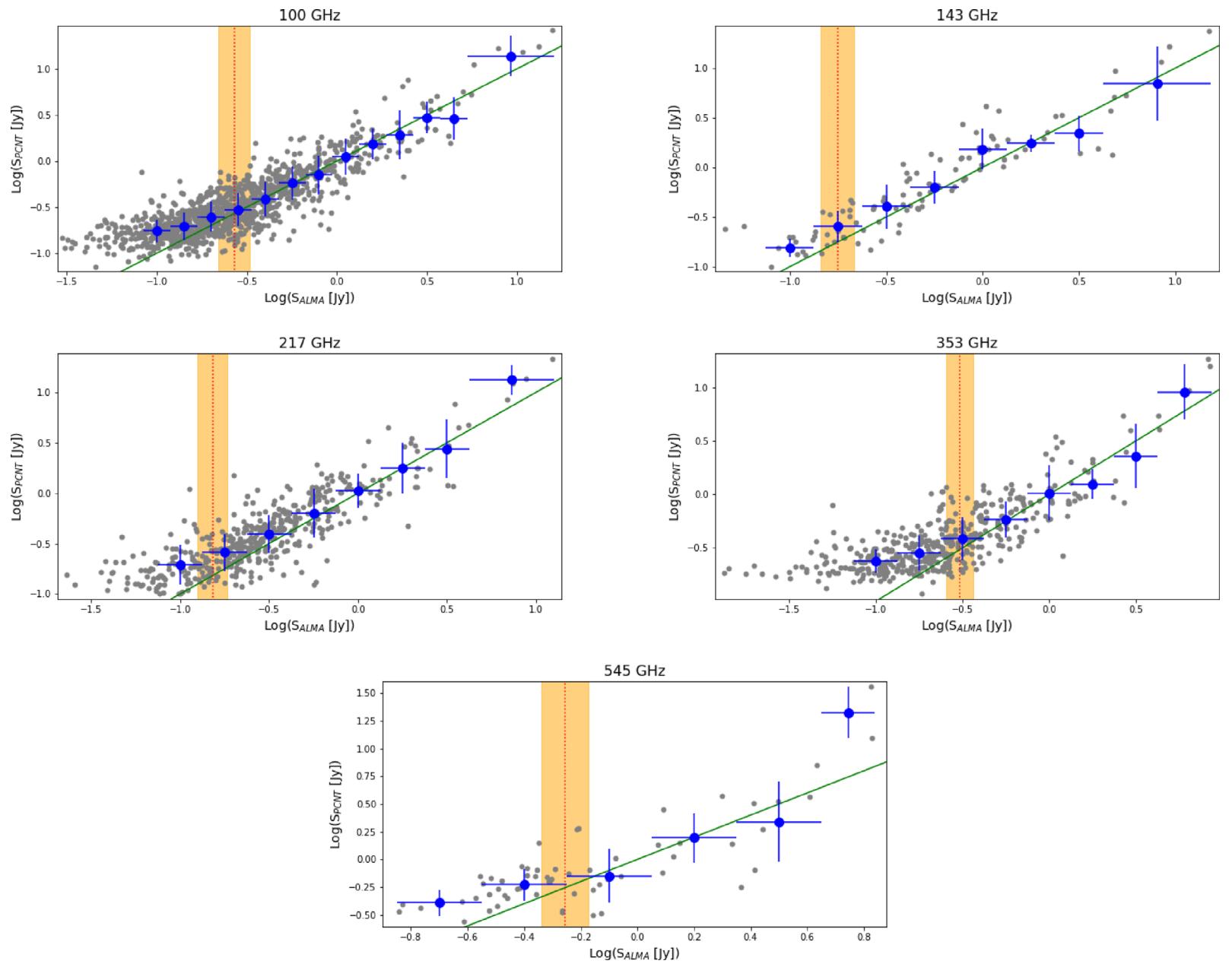

Figure 2. Mean values of $\log \left(S_{\mathrm{PCNT}}\right)$ (large blue circles with error bars) as a function of $\log \left(S_{\mathrm{ALMA}}\right)$ in bands $3,4,6,7$, and $8+9$. The tiny corrections for the slight differences between the effective frequencies of ALMA bands and those of the nearest Planck bands have been neglected except for bands 7,8 , and 9. Band $7(285 \mathrm{GHz})$ flux densities were extrapolated to $353 \mathrm{GHz}$ assuming the mean spectral index $\left(-0.45 ; S \propto v^{-0.45}\right)$ between bands 6 and 8 ; and bands 8 $(467 \mathrm{GHz})$ and $9(673 \mathrm{GHz})$ flux densities were extrapolated to $545 \mathrm{GHz}$ using the mean spectral index $(-0.62)$ between the two bands. The frequency intervals are small so that the results are weakly dependent on the choice for the spectral index. The horizontal bars correspond to the widths of log $\left(S_{\mathrm{ALMA}}\right)$ bins; and the vertical bars show the standard deviations around the mean values. The unbinned data points are represented by small grey dots. The vertical dotted red lines correspond to the PCCS2 90 per cent completeness limits with their uncertainties represented by the shaded orange bands. The green solid lines correspond to $S_{\mathrm{PCNT}}=S_{\mathrm{ALMA}}$.

The completeness of the PCNT catalogue at each frequency $\geq$ $100 \mathrm{GHz}$ was tested by looking at the fraction of ACC objects having PCNT counterparts with $\mathrm{S} / \mathrm{N} \geq 3$ as a function of the ALMA flux density. The results are shown in Table 2480.0 pt and Fig. 3. At $100,217,353$, and $545 \mathrm{GHz}$ our results are in excellent agreement with the estimates of the 90 per cent completeness limits given by Planck Collaboration XXVI (2016).

At $143 \mathrm{GHz}$, the ALMA data suggest a significantly higher PCNT limit, but with limited statistics (only 100 sources unevenly distributed among flux density bins, to be compared with 1069 sources at $100 \mathrm{GHz}, 455$ at $217 \mathrm{GHz}$, and 439 at $353 \mathrm{GHz}$ ). To assess the significance of the discrepancy we have performed 10000 simulations of Planck observations of the 100 ALMA calibrators. These simulations were carried out randomly extracting each source from a Gaussian distribution with mean value equal to its ALMA flux density and dispersion equal to the mean PCNT uncertainty of those with a PCNT counterpart $(\sim 36 \mathrm{mJy})$. A 90 percent completeness limit within the uncertainty of the PCCS2 value was found in 10 percent of the cases, implying that the discrepancy is only marginally significant. We have also checked whether the difference of the completeness limits may be due to the different photometric estimators used for the PCCS2 and the PCNT (although the two photometries were shown to be, on average, in good agreement by Planck Collaboration Int. LIV 2018). To this end, we have estimated the PCCS2 completeness levels in the same way as we did for the PCNT. The results did not change significantly.

At $857 \mathrm{GHz}$, the poor statistics (only four calibrators with ALMA band 10 flux density measurements have PCNT counterparts) hampers a reliable estimate. However, an indication that the highfrequency PCCS2 completeness limits are conservative was provided by the cross-match of the Herschel Astrophysical Terahertz Large Area Survey (H-ATLAS) with the PCCS2 catalogue (Maddox et al. 2018). These authors found PCCS 290 per cent completeness limits of $S_{\text {Herschel }}=650 \mathrm{mJy}$ at $857 \mathrm{GHz}(350 \mu \mathrm{m})$. For comparison, the PCCS2 estimate of the corresponding 90 per cent completeness limits is of $791 \mathrm{mJy}$. Using their catalogues, we further derived the 100 per cent PCCS2 completeness limits to be of $760 \mathrm{mJy}$ at this frequency.

These results indicate that, although the MTFX approach reaches, at fixed $\mathrm{S} / \mathrm{N}$, fainter flux densities than the single-frequency 
Table 2. PCCS2 90 percent completeness limits compared with the estimated PCNT 90 per cent and 100 per cent completeness limits, defined as the ALMA flux densities above which 90 per cent or 100 per cent of sources observed by ALMA have PCNT counterparts. Since there are too few sources observed in the ALMA band 10 to derive meaningful limits, we give the 90 per cent and 100 per cent limits derived by Maddox et al. (2018) from H-ATLAS data.

\begin{tabular}{lccccc}
\hline Planck v (GHz) & $\begin{array}{c}\text { PCCS2 90 per cent } \\
\text { completeness } \\
(\mathrm{mJy})\end{array}$ & $\begin{array}{c}\text { PCNT 90 per cent } \\
\text { completeness } \\
(\mathrm{mJy})\end{array}$ & $\begin{array}{c}\text { PCNT 100 per cent } \\
\text { completeness } \\
(\mathrm{mJy})\end{array}$ & $\begin{array}{c}\text { H-ATLAS 90 per cent } \\
\text { completeness } \\
(\mathrm{mJy})\end{array}$ & $\begin{array}{c}\text { H-ATLAS 100 per cent } \\
\text { completeness } \\
(\mathrm{mJy})\end{array}$ \\
\hline 100 & 269 & 266 & 562 & \\
143 & 177 & 312 & 562 & \\
217 & 152 & 170 & 316 & \\
353 & 304 & 296 & 1000 & & \\
545 & 555 & 597 & 1585 & & \\
857 & 791 & & & & 760 \\
\hline
\end{tabular}
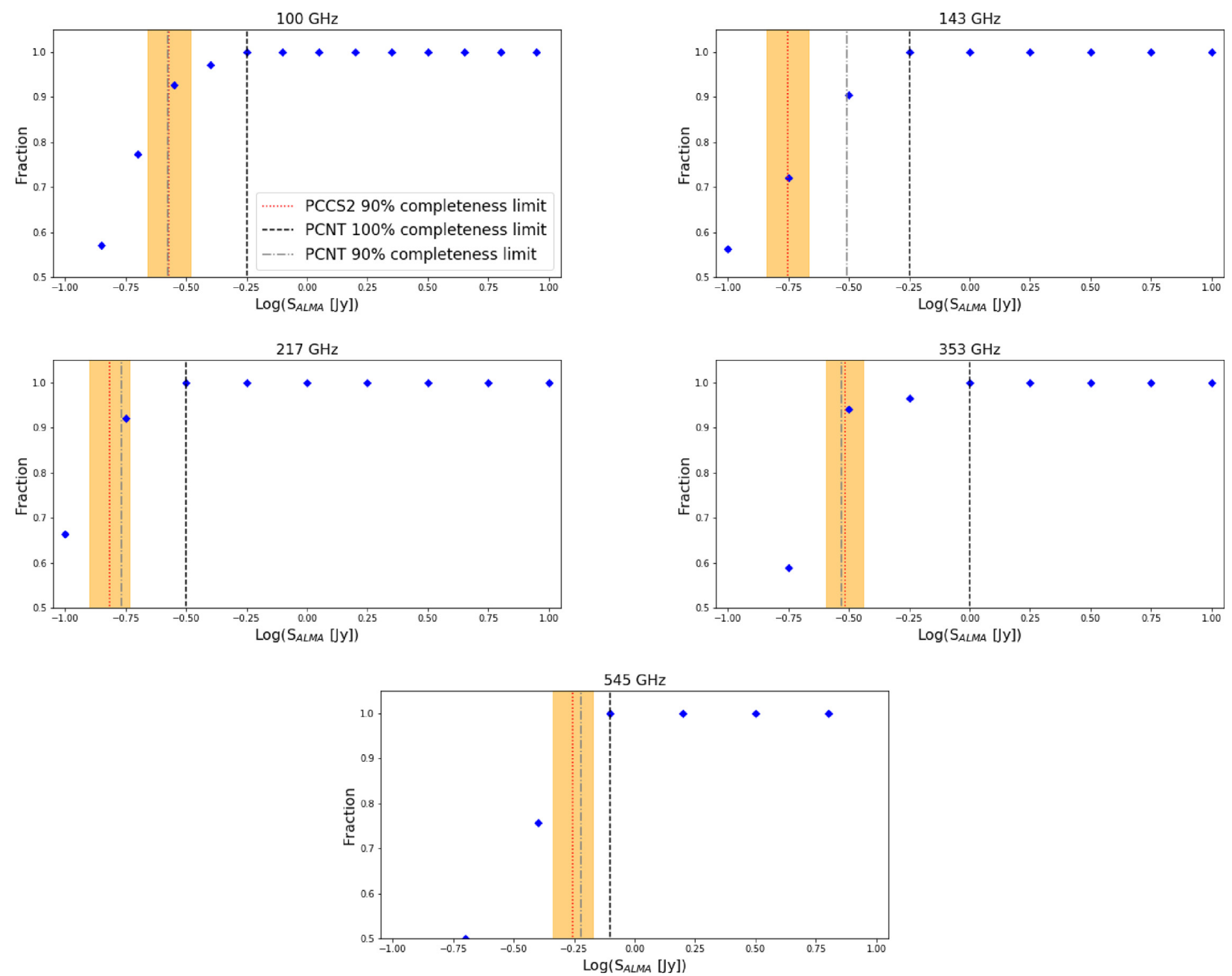

Figure 3. Fraction of ACC sources with PCNT counterparts at 100, 143, 217, 353, and $545 \mathrm{GHz}$ as a function of ALMA flux density in bands 3, 4, 6, 7, and $8+9$, respectively. The ALMA flux densities in the last two bands have been extrapolated to $545 \mathrm{GHz}$ adopting the mean spectral index of our sources between the two bands, i.e. $S \propto v^{-0.62}$. Band 7 flux densities have been extrapolated to $353 \mathrm{GHz}$ using the mean spectral index ( -0.45 ) between bands 6 and 8 . The vertical dashed black line and the dotted-dashed grey line show, at each frequency, the flux densities above which 100 per cent and 90 per cent of sources observed by ALMA have PCNT counterparts with $\mathrm{S} / \mathrm{N} \geq 3$. The dotted red lines show, for comparison, the PCCS 290 per cent completeness limits in the 'extragalactic zone' with their uncertainties (shaded orange bands). The number of sources observed by ALMA in band 10 is too small to allow meaningful estimates of the detection limits; see however the text. 

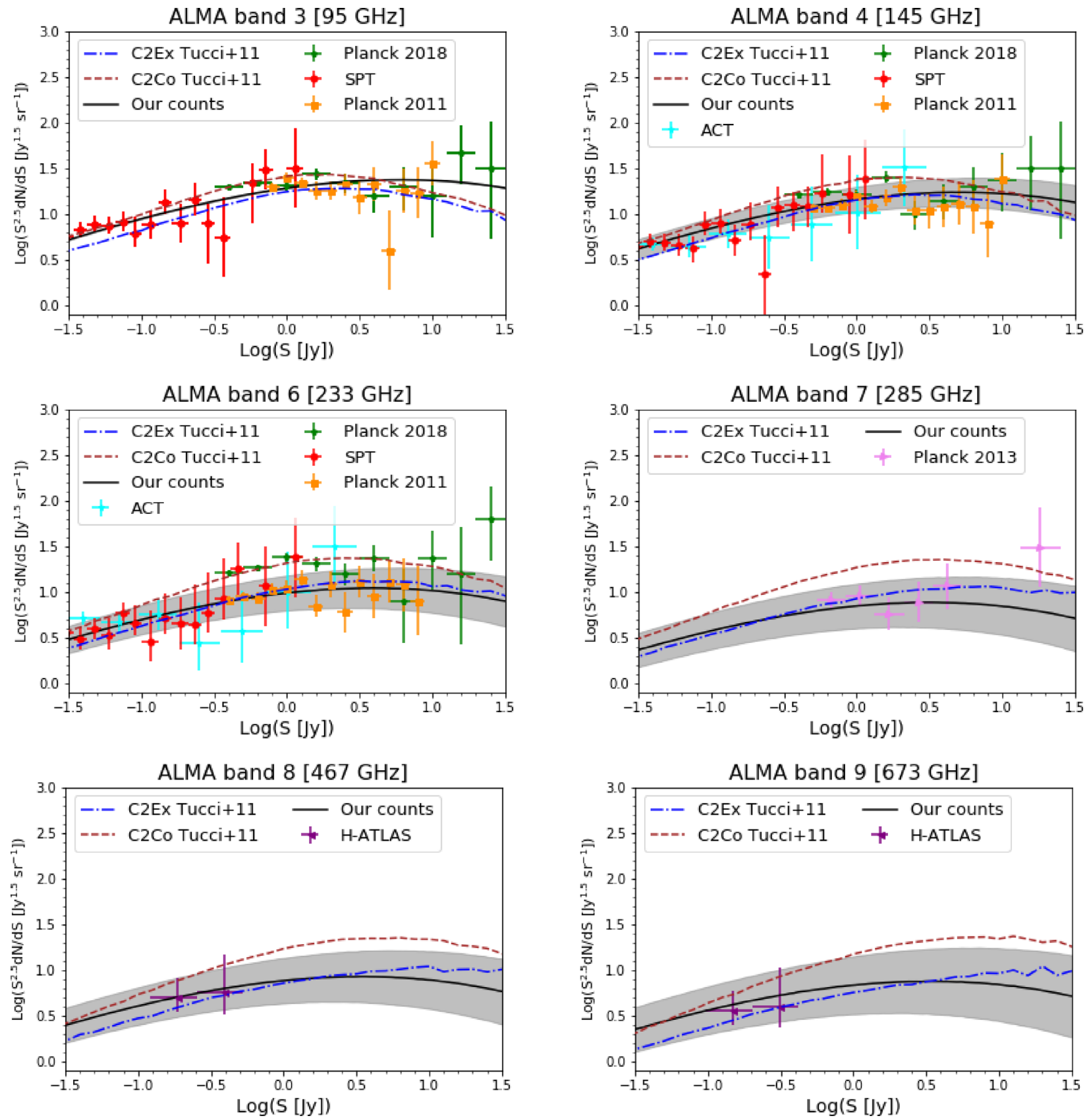

Figure 4. Euclidean normalized differential source counts of radio sources in the ALMA bands for which measurements for a substantial number of calibrators are available. The data are from Planck Collaboration XIII (2011), Mocanu et al. (2013, SPT), Planck Collaboration VII (2013), Marsden et al. (2014, ACT), and Planck Collaboration Int. LIV (2018). The bands 8 and 9 data points are our own estimates using table 1 of Negrello et al. (2017); the extrapolations of the $600 \mathrm{GHz}(500 \mu \mathrm{m})$ flux densities given there to the effective frequencies of these two bands were done assuming $S \propto v^{-0.62}$, i.e. using the mean spectral index between bands 8 and 9. The mean spectral index between bands 6 and $8(-0.45)$ was used to convert the Planck Collaboration VII (2013) counts from 353 to $285 \mathrm{GHz}$. The dotted-dashed blue line and the dashed brown line show two models by Tucci et al. (2011).

approach adopted for the PCCS2, and therefore the PCNT contains substantially more radio sources, ${ }^{6}$ the completeness limits remain essentially unchanged.

${ }^{6}$ The total number of PCCS2 sources at high frequencies is higher because it includes also dusty galaxies, while the PCNT contains, by construction, only radio sources.

\section{HIGH FREQUENCY NUMBER COUNTS OF RADIO SOURCES}

The combination of Planck (Planck Collaboration Int. LIV 2018) and SPT (Mocanu et al. 2013) data has provided an observational determination of radio source number counts at $\simeq 100 \mathrm{GHz}$ over a broad flux density range. An empirical description of the Euclidean normalized counts at this frequency is (solid black line in the top 
Table 3. Mean values of the log of the ratios of ALMA band 3 (characteristic frequency of $95 \mathrm{GHz}$ ) flux densities to those measured in the other ALMA bands and associated standard deviation, $\sigma$. By characteristic frequency of a band (second column), we mean the median frequency of the observations in such a band.

\begin{tabular}{lcccc}
\hline $\begin{array}{l}\text { Number of ALMA } \\
\text { band }\end{array}$ & $\begin{array}{c}\text { Characteristic } v \\
(\mathrm{GHz})\end{array}$ & $\begin{array}{c}\text { Number of } \\
\text { sources }\end{array}$ & $\left\langle\log \left(S_{95 \mathrm{GHz}} / S_{v}\right)\right\rangle$ & $\sigma$ \\
\hline 4 & 145 & 164 & 0.11 & 0.10 \\
6 & 233 & 859 & 0.25 & 0.14 \\
7 & 285 & 2102 & 0.37 & 0.17 \\
8 & 467 & 87 & 0.35 & 0.18 \\
9 & 673 & 58 & 0.41 & 0.23 \\
\hline
\end{tabular}

Table 4. Coefficients of the polynomial representations of the extrapolated Euclidean normalized differential counts of radio sources at the effective frequencies of 5 ALMA bands: $y=A \cdot x^{3}+B \cdot x^{2}+C \cdot x+D$, with $x=\log (S[\mathrm{Jy}])$ and $y=\log \left(S^{2.5} \mathrm{~d} N / \mathrm{d} S\left[\mathrm{Jy}^{1.5} \mathrm{sr}^{-1}\right]\right)$.

\begin{tabular}{lccccc}
\hline $\begin{array}{l}\text { Number of ALMA } \\
\text { band }\end{array}$ & $\begin{array}{c}\text { Characteristic } \\
(\mathrm{GHz})\end{array}$ & $A$ & $B$ & $C$ & $D$ \\
\hline 4 & 145 & -0.0166 & -0.128 & 0.207 & 1.160 \\
6 & 233 & -0.0160 & -0.133 & 0.175 & 0.988 \\
7 & 285 & -0.0159 & -0.136 & 0.151 & 0.846 \\
8 & 467 & -0.0159 & -0.135 & 0.159 & 0.883 \\
9 & 673 & -0.0157 & -0.134 & 0.156 & 0.833 \\
\hline
\end{tabular}

left panel of Fig. 4)

$y=-0.01684 \cdot x^{3}-0.1252 \cdot x^{2}+0.2264 \cdot x+1.282$,

where $x=\log (S[\mathrm{Jy}])$ and $y=\log \left(S^{2.5} \mathrm{~d} N / \mathrm{d} S\left[\mathrm{Jy}^{1.5} \mathrm{Sr}^{-1}\right]\right)$. We have exploited ALMA observations to extrapolate these counts to higher frequencies where direct survey data are increasingly poor. To this end, we have calculated the mean values and the dispersions of the $\log$ of the flux density ratios between band 3 (characteristic frequency of $95 \mathrm{GHz}$ ) and the ALMA higher frequency bands for which we have sufficient statistics. The results are shown in Table 3.

By convolving equation (1) with the appropriate distribution of flux density ratios, assumed to be a Gaussian with mean and standard deviation given by Table 3, we have obtained the counts in bands 4 , $6,7,8$, and 9 shown by the solid black lines in Fig. 4. The shaded grey bands represent the $1 \sigma$ uncertainties on the extrapolated counts. These number counts are available in a machine readable format in the website of the Italian node of the European ALMA Regional Centre (ARC, http://arc.ia2.inaf.it). Polynomial representations of the extrapolated counts are given in Table 4.

The extrapolated counts are fully consistent with the available data. The comparison with the two preferred models by Tucci et al. (2011), 'C2Co' and 'C2Ex', shows that the latter performs quite well at all frequencies while the former tends to overpredict the counts above $145 \mathrm{GHz}$.

\section{CONCLUSIONS}

We have presented a new catalogue of ALMA flux density measurements of radio sources, mostly blazars, used as flux density, bandpass response, amplitude, and phase visibility calibrators. The catalogue was built combining the ALMACAL catalogue published by Bonato et al. (2018) with the ALMA Calibrator Source Catalogue. It contains ALMA observations for 3364 bright, compact radio sources observed between 2011 May and 2018 July, for a total of 47115 observations in different bands and epochs. The catalogue is available online as Supporting Information and on the website of the Italian ARC (http://arc.ia2.inaf.it).
We have added redshifts found in the literature, available for 2245 (67 per cent) of the sources and a classification for all of them. The classification given in the 5th edition of the BZCAT (Massaro et al. 2009) was adopted for the 1391 objects listed there. The others were classified following Bonato et al. (2018).

The ALMA measurements were exploited to obtain the first external validation of the MTFX photometry presented in the new PCNT (Planck Collaboration Int. LIV 2018), to quantify its positional accuracy and to estimate its completeness limits.

We found good agreement between the ALMA and the MTFX photometry above the 90 percent completeness limits given by Planck Collaboration XXVI (2016). The dispersions around the mean MTFX/ALMA flux density ratios as a function of ALMA flux densities can be accounted for by variability which also explains the excess flux densities measured by Planck for the few brightest sources, most likely detected in a flaring phase. Below these limits, Planck measurements show clear signs of the Eddington bias.

The distribution of differences between ALMA and Planck positions peaks at $\sim 0.38$ arcmin and has a standard deviation $\sigma \simeq 0.64$ arcmin, confirming the Planck Collaboration XXVI (2016) conclusion that the PCCS2 positional accuracy is typically better than 1 arcmin. The distribution has however an extended tail reaching a few arcminutes. The extension of such tail slightly decreases if sources below the PCCS2 90 percent completeness limits are excluded.

An analysis of the fraction of ALMA calibrators with a PCNT counterpart having $\mathrm{S} / \mathrm{N} \geq 3$ at the nearest frequency as a function of the ALMA flux density has shown that the PCNT completeness limits are consistent with the PCCS2 ones at 100, 217, 353, and $545 \mathrm{GHz}$. The PCNT limit at $143 \mathrm{GHz}$ seems to be higher, but the difference is only marginally significant because of the poor statistics. We conclude that although the PCNT reaches fainter flux density levels than the PCCS2, the completeness limits do not change appreciably.

Finally, we have exploited the multifrequency ALMA observations to derive the distribution of flux density ratios between band 
3 and the higher frequency bands. These distributions have allowed us to estimate the counts in such bands, where direct measurements are limited or almost completely missing, by extrapolating the relatively well-determined $100 \mathrm{GHz}$ counts of radio sources. The results agree with the available data and are consistent with the C2Ex model by Tucci et al. (2011), while their C2Co model is disfavoured.

\section{ACKNOWLEDGEMENTS}

We thank the anonymous referee for a careful reading of the manuscript and many constructive comments. This paper and the AKF and KAFE development are part of the activities for the ALMA Re-Imaging Study approved in the framework of the 2016 ESO Call for Development Studies for ALMA Upgrade (PI: Massardi). The study acknowledges partial financial support by the Italian Ministero dell'Istruzione, Università e Ricerca through the grant 'Progetti Premiali 2012 -iALMA' (CUP C52I13000140001). $\mathrm{MB}, \mathrm{MM}$, and GDZ acknowledge support from INAF under PRIN SKA/CTA FORECaST. GDZ acknowledges support from ASI/INAF agreement no. 2014-024-R.1 for the Planck LFI Activity of Phase E2 and from the ASI/Physics Department of the university of Roma-Tor Vergata agreement no. 2016-24-H.0 for study activities of the Italian cosmology community. MN acknowledges support from the European Union's Horizon 2020 research and innovation programme under the Marie Skłodowska-Curie grant agreement no. 707601. DH thanks the Spanish MINECO for partial financial support under project AYA2015-64508-P and funding from the European Union's Horizon 2020 research and innovation programme (COMPET-05-2015) under grant agreement no. 687312 (RADIOFOREGROUNDS). LB and JGN acknowledge financial support from the I+D 2015 project AYA2015-65887$\mathrm{P}$ (MINECO/FEDER). JGN also acknowledges financial support from the Spanish MINECO for a 'Ramon y Cajal' fellowship (RYC2013-13256). This paper makes use of the following ALMA data: ADS/JAO.ALMA\#2011.0.00001.CAL. ALMA is a partnership of ESO (representing its member states), NSF (USA) and NINS (Japan), together with NRC (Canada), MOST and ASIAA (Taiwan), and KASI (Republic of Korea), in cooperation with the Republic of Chile. The Joint ALMA Observatory is operated by ESO, AUI/NRAO, and NAOJ.

\section{REFERENCES}

Bennett C. L. et al., 2013, ApJS, 208, 20

Bonato M. et al., 2018, MNRAS, 478, 1512

Eddington A. S., 1913, MNRAS, 73, 359

González-Nuevo J., Argüeso F., López-Caniego M., Toffolatti L., Sanz J. L., Vielva P., Herranz D., 2006, MNRAS, 369, 1603

Herranz D., Sanz J. L., 2008, IEEE J. Sel. Top. Signal Process., 2, 727

Herranz D., López-Caniego M., Sanz J. L., González-Nuevo J., 2009, MNRAS, 394, 510

Hogg D. W., Turner E. L., 1998, PASP, 110, 727

López-Caniego M., Herranz D., González-Nuevo J., Sanz J. L., Barreiro R. B., Vielva P., Argüeso F., Toffolatti L., 2006, MNRAS, 370, 2047

Maddox S. J. et al., 2018, ApJS, 236, 30

Marsden D. et al., 2014, MNRAS, 439, 1556

Massaro E., Giommi P., Leto C., Marchegiani P., Maselli A., Perri M., Piranomonte S., Sclavi S., 2009, A\&A, 495, 691

Mocanu L. M. et al., 2013, ApJ, 779, 61

Negrello M. et al., 2017, MNRAS, 465, 3558

Planck Collaboration XIII, 2011, A\&A, 536, A13

Planck Collaboration Int. VII, 2013, A\&A, 550, A133

Planck Collaboration Int. LIV, 2018, A\&A, 619, A94

Planck Collaboration XXVI, 2016, A\&A, 594, A26

Tucci M., Toffolatti L., de Zotti G., Martínez-González E., 2011, A\&A, 533, A57

\section{SUPPORTING INFORMATION}

Supplementary data are available at MNRAS online.

\section{ACC_catalogue.dat}

Please note: Oxford University Press is not responsible for the content or functionality of any supporting materials supplied by the authors. Any queries (other than missing material) should be directed to the corresponding author for the article.

This paper has been typeset from a $\mathrm{T}_{\mathrm{E}} \mathrm{X} / \mathrm{L} \mathrm{A} \mathrm{T} \mathrm{X}$ file prepared by the author. 